\title{
Differential prevalence and correlates of whole blood Epstein-Barr virus DNA between HIV-positive and HIV- negative men who have sex with men in Shanghai, China
}

\author{
R. PAN ${ }^{1,2} \dagger, X \cdot \mathrm{LIU}^{1} \dagger, \mathrm{S} . \mathrm{ZHOU}^{1}, \mathrm{Z} \cdot \mathrm{NING}^{3}, \mathrm{H} \cdot \mathrm{ZHENG}^{4}, \mathrm{M} \cdot \mathrm{GAO}^{1}, \mathrm{Y} \cdot \mathrm{DING}^{1}$, \\ W. $\mathrm{YAO}^{2}, \mathrm{X} . \mathrm{LIAO}^{2}$ AND N. HE ${ }^{1 *}$ \\ ${ }^{1}$ Department of Epidemiology, School of Public Health, and the Key Laboratory of Public Health Safety of \\ Ministry of Education, Fudan University, Shanghai, China \\ ${ }^{2}$ Hongkou District Center for Disease Control and Prevention, Shanghai, China \\ ${ }^{3}$ Shanghai Center for Disease Control and Prevention, Shanghai, China \\ ${ }^{4}$ Shanghai Piaoxue Cultural Media Limited, Shanghai, China
}

Received 14 January 2017; Final revision 29 March 2017; Accepted 25 April 2017; first published online 5 June 2017

\section{SUMMARY}

This cross-sectional study aimed to examine and compare prevalence and correlates of whole blood Epstein-Barr virus (EBV) DNA between HIV-positive and HIV-negative men who have sex with men (MSM). Five hundred and four HIV-positive MSM and 504 age-matched HIVnegative MSM were recruited from an HIV counseling and testing clinic in Shanghai, China from November 2014 to November 2015 and were administered with a face-to-face questionnaire interview. Whole blood EBV DNA was tested by nested polymerase chain reaction assays on $E B N A-1, E B N A-2$, and $L M P-1$ genes. The prevalence of whole blood EBV DNA was $56 \cdot 0 \%$ (95\% CI 51.7-60.3\%) among HIV-positive MSM and 26.0\% (95\% CI 22.4-30.0\%) among HIVnegative MSM. Whole blood EBV DNA positivity was significantly associated with HIV infection (adjusted odds ratio (aOR) 3.43, 95\% CI 2.58-4.57) and frequent intake of pickled, smoked, or salty food (aOR $1 \cdot 71,95 \%$ CI $1 \cdot 02-2 \cdot 86$ ) in the whole sample, and with $<200$ cells $/ \mu 1$ CD4 cell counts (aOR 1.79, 95\% CI 1.05-3.05) and pickled, smoked, or salty food intake (aOR $3 \cdot 14,95 \%$ CI 1·39-7.08) in HIV-positive group. HIV-infected MSM are at higher risk of active EBV replication than HIV-uninfected MSM, underscoring needs of surveillance and research on EBV-related carcinogenesis in this population.

Key words: Cancer, EBV, HIV, MSM, risk factors.

\section{INTRODUCTION}

Highly active antiretroviral therapy (HAART) or combination antiretroviral therapy (cART) has

\footnotetext{
* Author for correspondence: Dr N. He, Department of Epidemiology, School of Public Health, and the Key Laboratory of Public Health Safety of Ministry of Education, Fudan University, P.O. Box 289, 138 Yi Xue Yuan Road, Shanghai 200032, China.

(Email: nhe@fudan.edu.cn)

$\dagger$ Equally contributed first authors.
}

significantly increased life expectancy of people living with HIV/AIDS (PLWHA) in the past decades [1]. This has resulted in a shift in patterns of morbidities and mortalities in this population. A remarkable note is that both AIDS-defining cancers (ADCs) and non-AIDS-defining cancers (NADCs) are more often seen among PLWHA than among the general population [2-4]. PLWHA are at long-term status of immunodeficiency, immune activation and inflammation caused by HIV infection [5, 6], and have been observed to be at higher risks for both ADCs and 
NADCs than general population even after cART [79]. In addition to higher prevalence of infection with cancer-related viruses such as hepatitis $\mathrm{C}$ virus (HCV), human papilloma virus (HPV), Kaposi sarcoma associated herpes virus (KSHV) among PLWHA, higher prevalence of certain behavioral and psychological cancer risk factors such as tobacco use, heavy drinking and depression, etc. also plays important roles in carcinogenesis in this population. Thus, surveillance on cancer incidence and mortality as well as risk factors in this population are important for learning the disease burden and targeting people at high risk for cancer. Moreover, the associations between risk factors and carcinogenesis might be different in this immune compromised population, appealing for more detailed exploration.

The Epstein-Barr virus (EBV) is from the herpes virus family and persistently infects more than $90 \%$ of human adults. EBV infects B cells and epithelial cells with different mechanisms and replication patterns, and shows life-long latency after the initial lytic period [10]. Although the infection is ubiquitous and usually asymptomatic, EBV is constantly associated with certain types of human cancers including Hodgkin's lymphoma (HL) [11], non-Hodgkin's lymphoma (NHL) $[12,13]$ and nasopharyngeal cancer (NPC) [14, 15], among which NHL is one of the three ADCs. It is believed that genetic characteristics from both the viral and host sides, diet, behavioral, and environmental risk factors interplay in EBVassociated cancer development. In addition to EBV infection, risk factors for NPC include HPV infection, male, several dietary and behavioral factors such as salted fish intake and smoking, and family history of NPC [16, 17]; potential risk factors for HL include age, male, weakened immune system, and family history of the disease [18]; and risk factors for NHL vary by subtypes, including male, HIV infection, oncogene activation $(c-M Y C)$, malaria endemicity, family history, cigarette smoking, alcohol consumption, autoimmune diseases, and occupational factors $[13,19]$.

EBV DNA can be detected in both tumor tissues and blood samples from patients with EBV-related cancers, and circulating viral DNA level was proved to be an important biomarker in risk stratification, disease progression, and prognosis for NPC [15, 20, 21] and lymphoma [22, 23]. It has been reported that the EBV DNA were detected more often among HIV infected individuals than negative ones, suggesting a more active viral replication [24]. However, little has been explored for the association between nonviral risk factors and circulating EBV DNA in understanding risk and progression of EBV-related cancers especially in PLWHA.

Approximately 575000 people were living with HIV/AIDS in China at the end of 2015. Higher prevalence and mortality of cancers have already been observed in this population [25]. Additionally, homosexual transmission of HIV between men has been markedly increasing in recent years, reporting a HIV prevalence of $8 \%$ in 2015 [26]. High prevalence of HIV infection and risky behaviors made it an urgent need to have close observation on cancer development in this population.

We hypothesize that HIV infection and other potential risk factors of EBV-related cancers may be associated with EBV DNA replication. This study was thus performed to compare whole blood EBV DNA positivity between HIV-positive and HIVnegative men who have sex with men (MSM), as well as to identify potential correlates with EBV DNA positivity in Shanghai, China.

\section{METHODS}

\section{Study design}

All HIV-positive MSM and 1:1 age-matched $( \pm 5$ years) HIV-negative MSM were recruited from an HIV counseling and testing clinic in Shanghai from November 2014 to November 2015 and were administered with a questionnaire interview. The study was approved by the Institutional Review Board of Fudan University and written informed consent was obtained from each participant.

\section{Study participants}

Subjects who met the following inclusion criteria were enrolled: (1) male; (2) 18 years old or above; (3) can give oral or written informed consent in Mandarin; and (4) had oral or anal sex with men within the past 12 months.

\section{Data collection}

Participants were administered with a face-to-face questionnaire interview by trained and experienced health professionals to ascertain socio-demographic characteristics including age, residency, ethnic group, education level, marital status, and monthly income; and behavioral characteristics including lifetime 
homosexual behaviors, number of male sexual partners, tobacco smoking, alcohol drinking, dietary habits especially on unhealthy food intake, and history of illicit drug use. HIV-related clinical characteristics such as $\mathrm{CD}^{+} \mathrm{T}$ cell counts, diagnosis of AIDS and status of receiving ART were obtained from the Chinese National Information System for AIDS Prevention and Control (CNISAPC) given that all PLWHA in China have registered with and been routinely followed-up by CNISAPC.

Participants were asked to report their frequency of pickled, smoked, or salty food intake, and frequency of having fresh vegetables or fruits in the past 6 months. Frequencies were reported as 'never', 'occasionally', or 'frequently or daily'.

For the measurement of tobacco smoking, alcohol drinking and illicit drug use, the definitions were as following. Smoked in the past 6 months was defined as had smoked at least one cigarette every day during that period. Drank in the past 6 months was defined as had drunk alcohol at least once per week during the past 6 months. Types of alcohol consumed include beer, yellow wine, rice wine, Chinese white wine (a kind of distilled spirit), red wine, white wine, whisky, brandy, etc. Illicit drugs use in the past 6 months was defined as used methamphetamine, ecstasy, heroin, marijuana, opium, cocaine, sedatives, ketamine or poppers (amyl nitrites) during the past 6 months.

Participants were asked about sexual behaviors and the frequency of condom use. And the total number of sexual partners in life time was also collected.

\section{EBV DNA extraction, amplification, and sequencing}

For each participant, $5 \mathrm{ml}$ venous blood withdrawn using an EDTA anticoagulation sterilized tube were further aliquoted in both whole blood and plasma, respectively. EBV-DNA was extracted from $200 \mu 1$ whole blood in a nucleic acid-free room by High Pure Viral Nucleic Acid Kit (Roche, Germany) according to the manufacturer's instruction. The DNA was eluted into $50 \mu$ l elution buffer provided by the kit and stored at $-20^{\circ} \mathrm{C}$ until testing by nested polymerase chain reaction (PCR).

Nested PCR was performed to amplify the EBNA-1, EBNA-2, and LMP-1 genes of EBV. PCR reaction system was prepared in another nuclease-free room. The reaction mixture contained a final concentration of $1 \times \mathrm{PCR}$ buffer, $200 \mu \mathrm{M}$ of each dNTP, $0 \cdot 4$ $\mu \mathrm{M}$ of each primer, and $1.25 \mathrm{U}$ of Taq polymerase. Outer and inner primers used for EBNA-2,
EBNA-1, and LMP-1 genes in this study are shown in Table 1. About $1 \mu \mathrm{l}$ of DNA (25-30 ng) was added into the mixture with aerosol-resistant pipette tips to avoid cross-pollution between samples in the nucleic acid extraction room. PCR amplification was performed in a different room to avoid pollution. The integrity of the extracted DNA and the exclusion of PCR inhibitors in samples were confirmed by amplifying the human gene. The first amplification round included an initial denaturation step at $95^{\circ} \mathrm{C}$ for 5 min, followed by 30 cycles of denaturation at $95^{\circ} \mathrm{C}$ for $1 \mathrm{~min}$, primers annealing at $50^{\circ} \mathrm{C}$ (EBNA-2)/ $47{ }^{\circ} \mathrm{C}$ (EBNA-1) $/ 52^{\circ} \mathrm{C}($ LMP-1) for $1 \mathrm{~min}$, and an extension step at $72{ }^{\circ} \mathrm{C}$ for $1 \mathrm{~min}$, followed by the final extension step at $72{ }^{\circ} \mathrm{C}$ for $10 \mathrm{~min}$. For the second round, $1 \mu \mathrm{l}$ of the first round PCR product was used as template and amplified under the same conditions. The second round of amplification products were tested in $2 \%$ agarose gel. The positive PCR products were purified and sequenced by the Beijing Genomics Institute (BGI), Beijing, China. The participant with any one of the above three target genes detected was defined as positive for EBV DNA.

\section{Statistical analyses}

Statistical analyses were performed using R (https:// www.r-project.org/). The categorical variables were compared using $\chi^{2}$ test or Fisher's Exact test when appropriate. Unconditional logistic regression was used to estimate the odds ratios and their $95 \%$ confidence intervals. The selection of variables into multiple logistic regression models was based on both its significance in the univariate regression and prior knowledge (for socio-demographic characteristics and potential known risk factors). A two-sided $P$-value $<0.05$ is considered as statistically significant.

\section{RESULTS}

\section{Socio-demographic characteristics}

A total of 1008 participants including 504 HIVpositive and $504 \mathrm{HIV}$-negative MSM were included in the study. Among them, $81 \cdot 7 \%$ aged at $21-40$ years (range 18-69 years), 62.4\% were non-local Shanghai residents, $96.0 \%$ were ethnic Han, $66.8 \%$ received college or higher education, $76.5 \%$ were never married, and $50.9 \%$ reported to have monthly income more than 5000 RMB yuan ( US\$735). The two groups were comparable in distributions of 
Table 1. Primers for amplification

\begin{tabular}{|c|c|c|c|c|}
\hline Gene & Sequences & Position & Product size & Reference \\
\hline \multicolumn{5}{|c|}{ EBNA-2 } \\
\hline 2.a & AGGGATGCCTGGACACAA & $48810-48827$ & 600 & \multirow{6}{*}{ Aitken et al. $[27,28]$} \\
\hline 2.b & GTGCTGGTGCTGCTGGTGG & $49410-49391$ & & \\
\hline 2.c & TCTTGATAGGGATCCGCTAGGATA & $48839-48862$ & 497 & \\
\hline 2.d & ACCGTGGTTCTGGACTATCTGGATC & $49335-49311$ & & \\
\hline 2.e & AGACTTAGTTGATGCCCTAG & & 140 & \\
\hline $2 . f$ & CATGGTAGCCTTAGGACA & & & \\
\hline \multicolumn{5}{|c|}{ EBNA-1 } \\
\hline 1.a & GTAGAAGGCCATTTTTCCAC & $109151-109170$ & 609 & \multirow{4}{*}{ Wang et al. [29] } \\
\hline 1.b & CTCCATCGTCAAAGCTGCA & $109741-109759$ & & \\
\hline 1.c & AGATGACCCAGGAGAAGKCCCAAGC & $109266-109290$ & 308 & \\
\hline 1.d & CAAAGGGGAGACGACTCAATGGTGT & $109549-109573$ & & \\
\hline \multicolumn{5}{|c|}{ C } \\
\hline OF & AGACAGTGTGGCTAAGGGAGT & $168039-168059$ & $804 / 774$ & \multirow{4}{*}{ Van Kooij et al. } \\
\hline OR & CTACAACAAAACTGGTGGACT & $168843-168823$ & & \\
\hline IF & TGATTAGCTAAGGCATTCCCA & $168075-168095$ & $539 / 509$ & \\
\hline IR & TGCTCTCAAAACCTAGGCGCA & $168609-168589$ & & \\
\hline
\end{tabular}

residency, ethnicity, and marital status, but were significantly different in age, education level, and monthly income (Table 2).

\section{Lifetime homosexual behaviors}

As shown in Table 2, 95.4\% of the participants had homosexual behavior with a main male partner, $17 \cdot 2 \%$ with a male sex worker, and $79 \cdot 1 \%$ with a casual male partner in their lifetime. The majority $(95.8 \%)$ of the participants reported having had two or more sexual partners and $41 \cdot 6 \%$ having had $\geqslant 10$ partners so far. Compared with HIV-negative controls, HIV-positive MSM reported significantly higher proportion of homosexual activities including unprotected casual sex and having $\geqslant 5$ partners.

\section{Cancer-related factors}

The majority $(87 \cdot 5 \%)$ of the participants reported having had pickled, smoked, or salty food at least once in the past 6 months, and 7.5\% had such food frequently or daily. Also $27 \cdot 4 \%, 37 \cdot 9 \%$, and $30 \cdot 5 \%$ of participants reported smoking tobacco, drinking alcohol and illicit drug use in the past 6 months. Forty percent had depressive symptoms when measured with the CES-D (Center for Epidemiologic Studies Depression Scale). Significantly higher proportions of HIV-positive MSM reported fresh vegetables or fruits intake, smoking, drugs use, and depression than HIV-negative group, but a lower proportion consumed alcohol (Table 2).

\section{HIV infection-related characteristics}

Among the 504 HIV-positive MSM, 142 (28.2\%) had been diagnosed with AIDS, $300(59 \cdot 5 \%)$ were receiving ART, and $90(17 \cdot 9 \%)$ showed $\mathrm{CD}^{+} \mathrm{T}$ cell counts $<200$ cells/ $\mu 1$ at the time of interview.

\section{Prevalence and correlates of whole blood EBV DNA}

The whole blood EBV DNA positivity was $56.0 \%$ (95\% CI $51 \cdot 6-60 \cdot 2 \%$ ) for HIV-positive MSM and $26 \cdot 0 \%(95 \%$ CI $22 \cdot 4-30 \cdot 0 \%)$ for HIV-negatives $\left(\chi^{2}=\right.$ 93.53, $P<0 \cdot 001)$.

In the univariate logistic regression analyses for all participants, positivity of whole blood EBV DNA was inversely associated with levels of education (crude odds ratio $(\mathrm{cOR})=0 \cdot 55,95 \%$ CI $0 \cdot 38-0 \cdot 82)$, but was positively associated with aged above 40 years old $(\mathrm{cOR}=1 \cdot 72,95 \%$ CI $1 \cdot 21-2 \cdot 45)$, ever married (cOR $=1.43,95 \%$ CI $1 \cdot 07-1 \cdot 92$ ), ever had commercial sex with a male sex worker without consistent condom use in life time (cOR $=1 \cdot 59,95 \%$ CI $1 \cdot 09-2 \cdot 31)$, ever had sex with a casual male partner without consistent condom use in lifetime (cOR $=1 \cdot 55,95 \%$ CI $1 \cdot 13$ $2 \cdot 14$ ), had five or more male partners in life time $(\mathrm{cOR}=1 \cdot 53,95 \%$ CI $1 \cdot 12-2 \cdot 10)$, frequent or daily intake of pickled, smoked, or salty food in the past 6 months (cOR $=1 \cdot 98,95 \%$ CI $1 \cdot 23-3 \cdot 17)$, used illicit drugs in the past 6 months $(\mathrm{cOR}=1 \cdot 45,95 \% \mathrm{CI} 1 \cdot 10$ $1 \cdot 90)$, had depression symptoms ( $\mathrm{cOR}=1 \cdot 31,95 \% \mathrm{CI}$ $1 \cdot 01-1.69)$ and HIV infection ( $\mathrm{cOR}=3.62,95 \% \mathrm{CI}$ $2 \cdot 77-4 \cdot 72$ ) (Table 3). In the multiple logistic regression 
Table 2. Distribution and comparison of characteristics between HIV-positive and HIV-negative MSM in the study $(N=1008)$

\begin{tabular}{|c|c|c|c|c|c|c|c|c|}
\hline \multirow[b]{2}{*}{ Characteristics } & \multicolumn{2}{|c|}{ HIV negative } & \multicolumn{2}{|c|}{ HIV positive } & \multicolumn{2}{|c|}{ Total } & \multirow[b]{2}{*}{$\chi^{2}$} & \multirow[b]{2}{*}{$P$} \\
\hline & $n_{1}$ & $\%$ & $n_{2}$ & $\%$ & $n$ & $\%$ & & \\
\hline \multicolumn{9}{|l|}{ Socio-demographic characteristics } \\
\hline \multicolumn{9}{|l|}{ Age (years) } \\
\hline $18-20$ & 20 & $4 \cdot 0$ & 19 & $3 \cdot 8$ & 39 & $3 \cdot 9$ & $18 \cdot 205$ & $0 \cdot 003$ \\
\hline $21-30$ & 278 & $55 \cdot 1$ & 238 & $47 \cdot 2$ & 516 & $51 \cdot 1$ & & \\
\hline $31-40$ & 156 & $31 \cdot 0$ & 152 & $30 \cdot 2$ & 308 & $30 \cdot 6$ & & \\
\hline $41-50$ & 31 & $6 \cdot 1$ & 50 & $9 \cdot 9$ & 81 & $8 \cdot 0$ & & \\
\hline $51-60$ & 15 & $3 \cdot 0$ & 36 & $7 \cdot 1$ & 51 & $5 \cdot 1$ & & \\
\hline $61-69$ & 4 & $0 \cdot 8$ & 9 & $1 \cdot 8$ & 13 & $1 \cdot 3$ & & \\
\hline \multicolumn{9}{|l|}{ Permanent legal residency } \\
\hline Local & 201 & $39 \cdot 9$ & 178 & $35 \cdot 3$ & 379 & $37 \cdot 6$ & $2 \cdot 237$ & $0 \cdot 135$ \\
\hline Non-local & 303 & $60 \cdot 1$ & 326 & $64 \cdot 7$ & 629 & $62 \cdot 4$ & & \\
\hline \multicolumn{9}{|l|}{ Ethnic group } \\
\hline Han & 482 & $95 \cdot 6$ & 486 & $96 \cdot 4$ & 968 & $96 \cdot 0$ & $0 \cdot 614$ & $0 \cdot 433$ \\
\hline Other & 22 & $4 \cdot 4$ & 18 & $3 \cdot 6$ & 40 & $4 \cdot 0$ & & \\
\hline \multicolumn{9}{|l|}{ Level of education } \\
\hline Primary school & 3 & $0 \cdot 6$ & 7 & $1 \cdot 4$ & 10 & $1 \cdot 0$ & $18 \cdot 336$ & $<0 \cdot 001$ \\
\hline Middle school & 33 & $6 \cdot 6$ & 70 & $13 \cdot 9$ & 103 & $10 \cdot 2$ & & \\
\hline High school & 108 & $21 \cdot 4$ & 114 & $22 \cdot 6$ & 222 & $22 \cdot 0$ & & \\
\hline College or above & 360 & $71 \cdot 4$ & 313 & $62 \cdot 1$ & 673 & $66 \cdot 8$ & & \\
\hline \multicolumn{9}{|l|}{ Marital status } \\
\hline Never married & 402 & $79 \cdot 7$ & 369 & $73 \cdot 3$ & 771 & $76 \cdot 5$ & $5 \cdot 994$ & $0 \cdot 052$ \\
\hline Currently married & 75 & $14 \cdot 9$ & 99 & $19 \cdot 6$ & 174 & $17 \cdot 2$ & & \\
\hline Divorced/widowed & 27 & $5 \cdot 4$ & 36 & $7 \cdot 1$ & 63 & $6 \cdot 3$ & & \\
\hline \multicolumn{9}{|l|}{ Monthly income (Yuan, RMB) } \\
\hline$<1000$ & 39 & $7 \cdot 8$ & 49 & $9 \cdot 7$ & 88 & $8 \cdot 7$ & $23 \cdot 188$ & $<0.001$ \\
\hline $1000-2999$ & 47 & $9 \cdot 3$ & 83 & $16 \cdot 5$ & 130 & $12 \cdot 9$ & & \\
\hline $3000-4999$ & 126 & $25 \cdot 0$ & 151 & $30 \cdot 0$ & 277 & $27 \cdot 5$ & & \\
\hline$\geqslant 5000$ & 292 & $57 \cdot 9$ & 221 & $43 \cdot 8$ & 513 & $50 \cdot 9$ & & \\
\hline \multicolumn{9}{|l|}{ Lifetime homosexual behaviors } \\
\hline \multicolumn{9}{|l|}{ Ever had sex with a main male partner } \\
\hline No & 16 & $3 \cdot 2$ & 31 & $6 \cdot 2$ & 47 & $4 \cdot 6$ & $7 \cdot 120$ & $0 \cdot 028$ \\
\hline Yes, with consistent condom use & 37 & $7 \cdot 3$ & 25 & $5 \cdot 0$ & 62 & $6 \cdot 2$ & & \\
\hline Yes, without consistent condom use & 451 & $89 \cdot 5$ & 448 & $88 \cdot 9$ & 899 & $89 \cdot 2$ & & \\
\hline \multicolumn{9}{|c|}{ Ever had commercial sex with a male sex worker } \\
\hline No & 424 & $84 \cdot 1$ & 411 & $81 \cdot 5$ & 835 & $82 \cdot 8$ & $1 \cdot 254$ & $0 \cdot 534$ \\
\hline Yes, with consistent condom use & 23 & $4 \cdot 6$ & 25 & $5 \cdot 0$ & 48 & $4 \cdot 8$ & & \\
\hline Yes, without consistent condom use & 57 & $11 \cdot 3$ & 68 & $13 \cdot 5$ & 125 & $12 \cdot 4$ & & \\
\hline \multicolumn{9}{|l|}{ Ever had sex with a casual male partner } \\
\hline No & 136 & $27 \cdot 0$ & 74 & $14 \cdot 7$ & 210 & $20 \cdot 9$ & $24 \cdot 953$ & $<0.001$ \\
\hline Yes, with consistent condom use & 50 & $9 \cdot 9$ & 45 & $8 \cdot 9$ & 95 & $9 \cdot 4$ & & \\
\hline Yes, without consistent condom use & 318 & $63 \cdot 1$ & 385 & $76 \cdot 4$ & 703 & $69 \cdot 7$ & & \\
\hline \multicolumn{9}{|l|}{ Number of male partners } \\
\hline$<5$ & 145 & $28 \cdot 8$ & 81 & $16 \cdot 1$ & 226 & $22 \cdot 4$ & $23 \cdot 362$ & $<0 \cdot 001$ \\
\hline$\geqslant 5$ & 359 & $71 \cdot 2$ & 423 & $83 \cdot 9$ & 782 & $77 \cdot 6$ & & \\
\hline Cancer-related factors & & & & & & & & \\
\hline $\begin{array}{l}\text { Frequent or daily intake of pickled, sm } \\
\text { salty food in the past } 6 \text { months }\end{array}$ & & & & & & & & \\
\hline No & 471 & $93 \cdot 5$ & 461 & $91 \cdot 5$ & 932 & $92 \cdot 5$ & $1 \cdot 423$ & $0 \cdot 233$ \\
\hline Yes & 33 & $6 \cdot 5$ & 43 & $8 \cdot 5$ & 76 & $7 \cdot 5$ & & \\
\hline $\begin{array}{l}\text { Frequent or daily intake of fresh veget: } \\
\text { fruits in the past } 6 \text { months }\end{array}$ & & & & & & & & \\
\hline No & 179 & $35 \cdot 5$ & 123 & $24 \cdot 4$ & 302 & $30 \cdot 0$ & $14 \cdot 826$ & $<0 \cdot 001$ \\
\hline Yes & 325 & $64 \cdot 5$ & 381 & $75 \cdot 6$ & 706 & $70 \cdot 0$ & & \\
\hline
\end{tabular}


Table 2 (cont.)

\begin{tabular}{|c|c|c|c|c|c|c|c|c|}
\hline \multirow[b]{2}{*}{ Characteristics } & \multicolumn{2}{|c|}{ HIV negative } & \multicolumn{2}{|c|}{ HIV positive } & \multicolumn{2}{|c|}{ Total } & \multirow[b]{2}{*}{$\chi^{2}$} & \multirow[b]{2}{*}{$P$} \\
\hline & $n_{1}$ & $\%$ & $n_{2}$ & $\%$ & $n$ & $\%$ & & \\
\hline \multicolumn{9}{|c|}{ Smoked in the past 6 months } \\
\hline No & 385 & $76 \cdot 4$ & 347 & $68 \cdot 8$ & 732 & $72 \cdot 6$ & \multirow[t]{2}{*}{$7 \cdot 205$} & \multirow[t]{2}{*}{0.007} \\
\hline Yes & 119 & $23 \cdot 6$ & 157 & $31 \cdot 2$ & 276 & $27 \cdot 4$ & & \\
\hline \multicolumn{9}{|c|}{ Drank in the past 6 months } \\
\hline No & 289 & $57 \cdot 3$ & 337 & $66 \cdot 9$ & 626 & $62 \cdot 1$ & \multirow[t]{2}{*}{$9 \cdot 712$} & \multirow[t]{2}{*}{$0 \cdot 002$} \\
\hline Yes & 215 & $42 \cdot 7$ & 167 & $33 \cdot 1$ & 382 & $37 \cdot 9$ & & \\
\hline \multicolumn{9}{|c|}{ Used illicit drugs in the past 6 months } \\
\hline No & 383 & $76 \cdot 0$ & 318 & $63 \cdot 1$ & 701 & $69 \cdot 5$ & \multirow[t]{2}{*}{$19 \cdot 789$} & \multirow[t]{2}{*}{$<0.001$} \\
\hline Yes & 121 & $24 \cdot 0$ & 186 & $36 \cdot 9$ & 307 & $30 \cdot 5$ & & \\
\hline \multicolumn{9}{|c|}{ Depressive symptoms } \\
\hline No & 331 & $65 \cdot 7$ & 269 & $53 \cdot 4$ & 600 & $59 \cdot 5$ & \multirow[t]{2}{*}{$15 \cdot 828$} & \multirow[t]{2}{*}{$<0 \cdot 001$} \\
\hline Yes & 173 & $34 \cdot 3$ & 235 & $46 \cdot 6$ & 408 & $40 \cdot 5$ & & \\
\hline
\end{tabular}

analysis with adjustment for potential confounding variables, positivity of whole blood EBV DNA was positively associated with HIV infection (adjusted odds ratio $(\mathrm{aOR})=3.43,95 \%$ CI $2 \cdot 58-4 \cdot 57)$ and with frequent intake of pickled, smoked, or salty food $(\mathrm{aOR}=1 \cdot 71,95 \%$ CI 1·02-2·86) (Table 3$)$.

The associations were further examined stratified by status of HIV infection and were shown in Table 4. For HIV-infected MSM, 282 were tested positive for whole blood EBV DNA. Positivity of whole blood EBV DNA was positively associated with $\mathrm{CD}^{+}{ }^{+} \mathrm{T}$ cell counts $<200$ cells $/ \mu \mathrm{l}(\mathrm{aOR}=1 \cdot 79,95 \%$ CI $1 \cdot 05-$ 3.05 ) and with frequent intake of pickled, smoked, or salty food $(\mathrm{aOR}=3 \cdot 14,95 \%$ CI $1 \cdot 39-7 \cdot 08)$. For HIV-negative MSM, 131 were tested positive for whole blood EBV DNA and the positivity was only significantly associated with monthly income $(3000$ 4999 vs. $<1000 \mathrm{RMB}$ yuan: aOR $=3 \cdot 75,95 \% \mathrm{CI}$ $1 \cdot 21-11 \cdot 65 ; \geqslant 5000$ vs. $<1000$ RMB yuan: $\mathrm{aOR}=$ $3 \cdot 61,95 \%$ CI $1 \cdot 21-10 \cdot 71)$ in the multiple logistic regression model.

\section{DISCUSSION}

In this cross-sectional study, we reported a higher prevalence of whole blood EBV DNA among HIV-positive participants and its positive association with dietary factors and immune-suppressed status in a MSM population from Shanghai, China. These results suggested an active EBV replication status among PLWHA, and appealed for further explorations in the risk factors and their interaction in EBV-related carcinogenesis.
The prevalence of whole blood EBV DNA was found to be $56 \cdot 0 \% \quad(95 \%$ CI $51 \cdot 6-60 \cdot 2 \%)$ for HIV-positive MSM and 26.0\% (95\% CI 22.4-30.0\%) for HIV-negative MSM in this study. Among limited literature in Chinese PLWHA, Wu et al. reported an overall prevalence of peripheral EBV DNA as $32 \cdot 3 \%$ in 257 HIV/AIDS patients from a hospital in Shenzhen [31]. Wang et al. reported higher prevalence among 73 HIV-infected participants when compared with 80 healthy controls in Henan $(35 \cdot 6 \%$ vs. $5 \cdot 0 \%)$ [32]. The prevalence for HIV-infected people found in the current study was higher than the above mentioned studies, probably because it targeted HIV-positive MSM population specifically. Evidence from a study performed in Amsterdam showed a prevalence of $67 \%$ for EBV DNA from peripheral blood among HIV-positive MSM, higher than the prevalence of $39 \%$ among HIV-negative MSM and the prevalence of $6 \%$ among HIV-negative heterosexual men [24]. Our results added to the evidence that HIV-positive homosexual men were at higher risk of having positive whole blood EBV DNA.

The higher prevalence of EBV DNA among PLWHA indicated that people with suppressed immune system were at higher risk for active EBV viral replication, which was also suggested by our finding that having $\mathrm{CD} 4^{+} \mathrm{T}$ cell counts $<200 \mathrm{cells} / \mathrm{ul}$ were positively associated with detectable EBV DNA. Wu's study also found higher EBV DNA prevalence among inpatients who were at advanced stage of AIDS than outpatients (44.0\% vs. $25 \cdot 9 \%, \chi^{2}$ $=8.7605, P<0.005)$ [31]. Chronic HIV infection causes antigen simulation of $\mathrm{B}$ cells, breaks the 
Table 3. Association between characteristics and whole blood EBV DNA positivity among MSM in the study

\begin{tabular}{|c|c|c|c|c|c|c|}
\hline \multirow[b]{2}{*}{ Characteristics } & \multicolumn{2}{|c|}{$\begin{array}{l}\text { EBV DNA } \\
\text { positivity }\end{array}$} & \multirow{2}{*}{$\begin{array}{l}\text { Crude OR } \\
(95 \% \mathrm{CI})\end{array}$} & \multirow[b]{2}{*}{$P$-value } & \multirow{2}{*}{$\begin{array}{l}\text { Adjusted } \mathrm{OR}^{\mathrm{a}} \\
(95 \% \mathrm{CI})\end{array}$} & \multirow[b]{2}{*}{$P$-value } \\
\hline & $n$ & $\%$ & & & & \\
\hline \multicolumn{7}{|l|}{ Age (years) } \\
\hline$\leqslant 40$ & 337 & $39 \cdot 0$ & $1 \cdot 00$ & & $1 \cdot 00$ & \\
\hline$>40$ & 76 & $52 \cdot 4$ & $1 \cdot 72(1 \cdot 21-2 \cdot 45)$ & 0.003 & $1 \cdot 20(0 \cdot 76-1 \cdot 89)$ & $0 \cdot 430$ \\
\hline \multicolumn{7}{|l|}{ Permanent legal residency } \\
\hline Local & 157 & $41 \cdot 4$ & $1 \cdot 00$ & & $1 \cdot 00$ & \\
\hline Non-local & 256 & $40 \cdot 7$ & $0 \cdot 97(0 \cdot 75-1 \cdot 26)$ & $0 \cdot 821$ & $0 \cdot 86(0 \cdot 64-1 \cdot 15)$ & $0 \cdot 310$ \\
\hline \multicolumn{7}{|l|}{ Ethnic group } \\
\hline Han & 399 & $41 \cdot 2$ & $1 \cdot 00$ & & $1 \cdot 00$ & \\
\hline Other & 14 & $35 \cdot 0$ & $0 \cdot 77(0 \cdot 40-1 \cdot 49)$ & 0.434 & $0 \cdot 82(0 \cdot 41-1 \cdot 66)$ & 0.584 \\
\hline \multicolumn{7}{|l|}{ Level of education } \\
\hline Middle school or below & 61 & $54 \cdot 0$ & $1 \cdot 00$ & & $1 \cdot 00$ & \\
\hline High school or above & 352 & $39 \cdot 3$ & $0 \cdot 55(0 \cdot 38-0 \cdot 82)$ & $0 \cdot 003$ & $0 \cdot 84(0 \cdot 53-1 \cdot 34)$ & $0 \cdot 457$ \\
\hline \multicolumn{7}{|l|}{ Marital status } \\
\hline Never married & 300 & $38 \cdot 9$ & $1 \cdot 00$ & & $1 \cdot 00$ & \\
\hline Ever married & 113 & $47 \cdot 7$ & $1.43(1.07-1.92)$ & $0 \cdot 017$ & $1 \cdot 11(0 \cdot 76-1 \cdot 61)$ & 0.592 \\
\hline \multicolumn{7}{|l|}{ Monthly income (Yuan, RMB) } \\
\hline$<1000$ & 37 & $42 \cdot 0$ & $1 \cdot 00$ & & $1 \cdot 00$ & \\
\hline 1000-2999 & 62 & $47 \cdot 7$ & $1 \cdot 26(0 \cdot 73-2 \cdot 17)$ & $0 \cdot 412$ & $1 \cdot 10(0 \cdot 61-2 \cdot 01)$ & $0 \cdot 747$ \\
\hline $3000-4999$ & 122 & $44 \cdot 0$ & $1.09(0.67-1.76)$ & 0.742 & $1 \cdot 13(0 \cdot 66-1 \cdot 93)$ & 0.658 \\
\hline$\geqslant 5000$ & 192 & $37 \cdot 4$ & $0 \cdot 82(0 \cdot 52-1 \cdot 31)$ & $0 \cdot 410$ & $1.04(0.63-1.73)$ & $0 \cdot 876$ \\
\hline \multicolumn{7}{|l|}{$\begin{array}{l}\text { Ever had commercial sex with a male sex } \\
\text { worker in lifetime }\end{array}$} \\
\hline No & 326 & $39 \cdot 0$ & $1 \cdot 00$ & & $1 \cdot 00$ & \\
\hline Yes, with consistent condom use & 24 & $50 \cdot 0$ & $1 \cdot 56(0 \cdot 87-2 \cdot 80)$ & $0 \cdot 134$ & $1.59(0.85-2 \cdot 96)$ & $0 \cdot 147$ \\
\hline Yes, without consistent condom use & 63 & $50 \cdot 4$ & $1 \cdot 59(1 \cdot 09-2 \cdot 31)$ & $0 \cdot 016$ & $1 \cdot 26(0 \cdot 83-1 \cdot 91)$ & $0 \cdot 271$ \\
\hline \multicolumn{7}{|l|}{$\begin{array}{l}\text { Ever had sex with a casual male partner } \\
\text { in lifetime }\end{array}$} \\
\hline No & 71 & $33 \cdot 8$ & $1 \cdot 00$ & & $1 \cdot 00$ & \\
\hline Yes, with consistent condom use & 31 & $32 \cdot 6$ & $0 \cdot 95(0 \cdot 57-1 \cdot 59)$ & $0 \cdot 840$ & $0 \cdot 80(0 \cdot 45-1 \cdot 41)$ & $0 \cdot 435$ \\
\hline Yes, without consistent condom use & 311 & $44 \cdot 2$ & $1 \cdot 55(1 \cdot 13-2 \cdot 14)$ & 0.007 & $1.09(0.74-1.61)$ & 0.654 \\
\hline \multicolumn{7}{|l|}{ Number of male partners in lifetime } \\
\hline$<5$ & 75 & $33 \cdot 2$ & $1 \cdot 00$ & & $1 \cdot 00$ & \\
\hline$\geqslant 5$ & 338 & $43 \cdot 2$ & $1 \cdot 53(1 \cdot 12-2 \cdot 10)$ & $0 \cdot 007$ & $1 \cdot 09(0 \cdot 75-1 \cdot 58)$ & $0 \cdot 645$ \\
\hline \multicolumn{7}{|l|}{$\begin{array}{l}\text { Frequent or daily intake of pickled, } \\
\text { smoked, } \\
\text { or salty food in the past } 6 \text { months }\end{array}$} \\
\hline No & 370 & $39 \cdot 7$ & $1 \cdot 00$ & & $1 \cdot 00$ & \\
\hline Yes & 43 & $56 \cdot 6$ & $1 \cdot 98(1 \cdot 23-3 \cdot 17)$ & 0.005 & $1 \cdot 71(1 \cdot 02-2 \cdot 86)$ & 0.044 \\
\hline \multicolumn{7}{|l|}{$\begin{array}{l}\text { Frequent or daily intake of fresh } \\
\text { vegetables } \\
\text { or fruits in the past } 6 \text { months }\end{array}$} \\
\hline No & 129 & $42 \cdot 7$ & $1 \cdot 00$ & & 1.00 & \\
\hline Yes & 284 & $40 \cdot 2$ & $0 \cdot 90(0 \cdot 69-1 \cdot 19)$ & $0 \cdot 462$ & $0 \cdot 78(0 \cdot 57-1 \cdot 05)$ & $0 \cdot 102$ \\
\hline \multicolumn{7}{|l|}{ Smoked in the past 6 months } \\
\hline No & 287 & $39 \cdot 2$ & $1 \cdot 00$ & & $1 \cdot 00$ & \\
\hline Yes & 126 & $45 \cdot 7$ & $1 \cdot 30(0 \cdot 99-1 \cdot 72)$ & 0.064 & $1.03(0.75-1 \cdot 42)$ & $0 \cdot 849$ \\
\hline \multicolumn{7}{|l|}{ Drunk in the past 6 months } \\
\hline No & 257 & $41 \cdot 1$ & $1 \cdot 00$ & & $1 \cdot 00$ & \\
\hline Yes & 156 & $40 \cdot 8$ & $0.99(0.77-1.28)$ & 0.946 & $1.08(0.80-1 \cdot 44)$ & 0.628 \\
\hline \multicolumn{7}{|l|}{ Used illicit drugs in the past 6 months } \\
\hline No & 268 & $38 \cdot 2$ & 1 & & $1 \cdot 00$ & \\
\hline Yes & 145 & $47 \cdot 2$ & $1 \cdot 45(1 \cdot 10-1 \cdot 90)$ & 0.008 & $1.26(0.93-1 \cdot 71)$ & $0 \cdot 136$ \\
\hline
\end{tabular}


Table 3 (cont.)

\begin{tabular}{|c|c|c|c|c|c|c|}
\hline \multirow[b]{2}{*}{ Characteristics } & \multicolumn{2}{|c|}{$\begin{array}{l}\text { EBV DNA } \\
\text { positivity }\end{array}$} & \multirow{2}{*}{$\begin{array}{l}\text { Crude OR } \\
(95 \% \mathrm{CI})\end{array}$} & \multirow[b]{2}{*}{$P$-value } & \multirow{2}{*}{$\begin{array}{l}\text { Adjusted } \mathrm{OR}^{\mathrm{a}} \\
(95 \% \mathrm{CI})\end{array}$} & \multirow[b]{2}{*}{$P$-value } \\
\hline & $n$ & $\%$ & & & & \\
\hline \multicolumn{7}{|c|}{ Depressive symptoms } \\
\hline No & 230 & $38 \cdot 3$ & $1 \cdot 00$ & & $1 \cdot 00$ & \\
\hline Yes & 183 & $44 \cdot 9$ & $1 \cdot 31(1 \cdot 01-1 \cdot 69)$ & 0.039 & $1 \cdot 08(0 \cdot 82-1 \cdot 43)$ & $0 \cdot 580$ \\
\hline \multicolumn{7}{|c|}{ HIV infection status } \\
\hline Negative & 131 & $26 \cdot 0$ & $1 \cdot 00$ & & $1 \cdot 00$ & \\
\hline Positive & 282 & $56 \cdot 0$ & $3 \cdot 62(2 \cdot 77-4 \cdot 72)$ & $<0 \cdot 001$ & $3 \cdot 43(2 \cdot 58-4 \cdot 57)$ & $<0 \cdot 001$ \\
\hline
\end{tabular}

${ }^{a}$ Adjusted for all the other variables in the table.

equilibrium that newly infected and differentiating B cells were controlled by cytotoxic $\mathrm{T}$ cell responses, and thus leads to uncontrolled lymphoproliferation and cancer development [12]. It has been observed that the standardized incidence rates for most NADCs were greater than doubled in HIV population, and lower $\mathrm{CD}^{+} \mathrm{T}$ cells and taking HAART were found to be two major risk factors [33]. Moreover, it has been believed that the EBV DNA load and HIV viremia are correlated [34] and could serve as the diagnostic and prognostic marker for lymphoma in HIV-infected patients [35-38]. Based on these evidence and our finding, we suggest closer surveillance of EBV-related cancer incidence, known risk factors, as well as biomarkers including EBV DNA especially among PLWHA with lower CD4 counts or with HIV viremia.

One major finding of the current study was that participants who frequently consume pickled, smoked, or salty food were at higher risk of having positive whole blood EBV DNA. Among known risk factors for NPC, salted fish intake was a well-established one and was firstly reported in Chinese population [39, 40]. Chinese-style salted fish was defined as Group I carcinogen by IARC (International Agency for Research on Cancer) since high levels of NDMA (N-nitrosodimethylamine) was generated during making the fish. In this study, we were the first to investigate participants' habit of pickled, smoked, or salty food intake, which was a broad range of food processed using salt or other sources, and found a positive association with circulating EBV DNA in MSM. In our observation, the association was only significant among HIV-positive MSM in stratified analyses. The possible explanations for the heterogeneity of the association include that HIV infection could be a stronger risk factor for active
EBV replication, making those under immunosuppressed condition more vulnerable to other potential cancer risk factors to play a role. This is consistent with our observation that lower CD4 $\mathrm{T}$ cell counts also showed to be a risk factor. Once confirmed, the association between pickled, smoked or salty food and active EBV replication would suggest a population at higher risk of EBV-related cancers for intervention and cancer prevention.

There are several limitations in the current study. First, by nature of a cross-sectional design, we may only describe the distribution of risk factors and associations identified in this study can be further investigated using other study designs. However, the associations found between positive whole blood EBV DNA and HIV infection and frequent pickled, smoked, or salty food intake still calls attention not only because it was the first time examining the association in this population, but also for the strong associations discovered. Second, residual confounding may be present and partly explain the association we found between EBV DNA and cancer risk factors. For example, HPV infection is a risk factor for NPC and has not been measured in this study. We collected information on sexual behaviors as a proxy, since HPV infection is associated with active and unsafe sex. A more comprehensive collection of information on exposures for known risk factors such as viral co-infection is needed in future research. Third, most of the exposure measurements from this study were based on self-reported data. Thus the information bias may exist especially for the validity of answers to sensitive questions such as on sexual behaviors and illicit drug use. Also, the measurements on tobacco and alcohol use, dietary patterns, and quantities of food can be improved for better estimates of the associations. However, the validity of the major finding on HIV infection and unhealthy food intake 
Table 4. Separate multiple logistic regression analyses of associates with whole blood EBV DNA positivity within the stratum of HIV-positive and HIV-negative MSM, respectively

\begin{tabular}{|c|c|c|c|c|c|c|c|c|}
\hline \multirow[b]{3}{*}{ Characteristics } & \multicolumn{4}{|c|}{ HIV positive } & \multicolumn{4}{|c|}{ HIV negative } \\
\hline & \multicolumn{2}{|c|}{$\begin{array}{l}\text { EBV DNA } \\
\text { positivity }\end{array}$} & \multirow{2}{*}{$\begin{array}{l}\text { Adjusted OR }{ }^{\mathrm{a}} \\
(95 \% \mathrm{CI})\end{array}$} & \multirow[b]{2}{*}{$P$-value } & \multicolumn{2}{|c|}{$\begin{array}{l}\text { EBV DNA } \\
\text { positivity }\end{array}$} & \multirow{2}{*}{$\begin{array}{l}\text { Adjusted } \mathrm{OR}^{\mathrm{a}} \\
(95 \% \mathrm{CI})\end{array}$} & \multirow[b]{2}{*}{$P$-value } \\
\hline & $n$ & $\%$ & & & $n$ & $\%$ & & \\
\hline \multicolumn{9}{|l|}{ Age (years) } \\
\hline$\leqslant 40$ & 224 & $54 \cdot 8$ & 1.00 & & 113 & $24 \cdot 9$ & $1 \cdot 00$ & \\
\hline$>40$ & 58 & $61 \cdot 1$ & $1 \cdot 12(0 \cdot 62-2 \cdot 00)$ & $0 \cdot 713$ & 18 & $36 \cdot 0$ & $1 \cdot 27(0 \cdot 61-2 \cdot 66)$ & $0 \cdot 520$ \\
\hline \multicolumn{9}{|l|}{ Permanent legal residency } \\
\hline Local & 101 & $56 \cdot 7$ & $1 \cdot 00$ & & 56 & $27 \cdot 9$ & $1 \cdot 00$ & \\
\hline Non-local & 181 & $55 \cdot 5$ & $0 \cdot 91(0 \cdot 60-1 \cdot 36)$ & $0 \cdot 629$ & 75 & $24 \cdot 8$ & $0 \cdot 77(0 \cdot 50-1 \cdot 19)$ & $0 \cdot 240$ \\
\hline \multicolumn{9}{|l|}{ Ethnic group } \\
\hline Han & 275 & $56 \cdot 6$ & $1 \cdot 00$ & & 124 & $25 \cdot 7$ & $1 \cdot 00$ & \\
\hline Other & 7 & $38 \cdot 9$ & $0 \cdot 55(0 \cdot 20-1 \cdot 46)$ & $0 \cdot 228$ & 7 & $31 \cdot 8$ & $1 \cdot 46(0 \cdot 55-3 \cdot 86)$ & $0 \cdot 451$ \\
\hline \multicolumn{9}{|l|}{ Level of education } \\
\hline Middle school or below & 47 & $61 \cdot 0$ & $1 \cdot 00$ & & 14 & 38.9 & $1 \cdot 00$ & \\
\hline High school or above & 235 & $55 \cdot 0$ & $0 \cdot 86(0 \cdot 49-1 \cdot 53)$ & $0 \cdot 617$ & 117 & $25 \cdot 0$ & $0 \cdot 66(0 \cdot 30-1 \cdot 47)$ & $0 \cdot 313$ \\
\hline \multicolumn{9}{|l|}{ Marital status } \\
\hline Never married & 205 & $55 \cdot 6$ & $1 \cdot 00$ & & 95 & $23 \cdot 6$ & $1 \cdot 00$ & \\
\hline Ever married & 77 & $57 \cdot 0$ & $0 \cdot 88(0 \cdot 53-1 \cdot 45)$ & $0 \cdot 607$ & 36 & $35 \cdot 3$ & $1 \cdot 46(0 \cdot 83-2 \cdot 57)$ & $0 \cdot 190$ \\
\hline \multicolumn{9}{|c|}{ Monthly income (Yuan, RMB) } \\
\hline$<1000$ & 33 & $67 \cdot 3$ & 1.00 & & 4 & $10 \cdot 3$ & 1.00 & \\
\hline 1000-2999 & 51 & $61 \cdot 4$ & $0 \cdot 80(0 \cdot 36-1 \cdot 76)$ & 0.574 & 11 & $23 \cdot 4$ & $2 \cdot 42(0 \cdot 68-8 \cdot 63)$ & $0 \cdot 175$ \\
\hline $3000-4999$ & 85 & $56 \cdot 3$ & $0 \cdot 70(0 \cdot 34-1 \cdot 44)$ & $0 \cdot 336$ & 37 & $29 \cdot 4$ & $3.75(1.21-11 \cdot 65)$ & $0 \cdot 022$ \\
\hline$\geqslant 5000$ & 113 & $51 \cdot 1$ & $0 \cdot 57(0 \cdot 29-1 \cdot 14)$ & $0 \cdot 110$ & 79 & $27 \cdot 1$ & $3 \cdot 61(1 \cdot 21-10 \cdot 71)$ & $0 \cdot 021$ \\
\hline \multicolumn{9}{|c|}{$\begin{array}{l}\text { Frequent or daily intake of pickled, smoked, } \\
\text { or salty food in the past } 6 \text { months }\end{array}$} \\
\hline No & 247 & $53 \cdot 6$ & $1 \cdot 00$ & & 123 & $26 \cdot 1$ & $1 \cdot 00$ & \\
\hline Yes & 35 & $81 \cdot 4$ & $3 \cdot 14(1 \cdot 39-7 \cdot 08)$ & $0 \cdot 006$ & 8 & $24 \cdot 2$ & $0 \cdot 87(0 \cdot 37-2 \cdot 04)$ & 0.749 \\
\hline \multicolumn{9}{|c|}{$\begin{array}{l}\text { Frequent or daily intake of fresh vegetables } \\
\text { or fruits in the past } 6 \text { months }\end{array}$} \\
\hline No & 77 & $62 \cdot 6$ & $1 \cdot 00$ & & 52 & $29 \cdot 1$ & $1 \cdot 00$ & \\
\hline Yes & 205 & $53 \cdot 8$ & $0 \cdot 75(0 \cdot 48-1 \cdot 16)$ & $0 \cdot 195$ & 79 & $24 \cdot 3$ & $0 \cdot 78(0 \cdot 50-1 \cdot 20)$ & $0 \cdot 250$ \\
\hline \multicolumn{9}{|l|}{ Smoked in the past 6 months } \\
\hline No & 186 & $53 \cdot 6$ & $1 \cdot 00$ & & 101 & $26 \cdot 2$ & $1 \cdot 00$ & \\
\hline Yes & 96 & $61 \cdot 1$ & $1 \cdot 18(0 \cdot 77-1 \cdot 81)$ & 0.437 & 30 & $25 \cdot 2$ & $0 \cdot 85(0 \cdot 51-1 \cdot 43)$ & 0.542 \\
\hline \multicolumn{9}{|l|}{ Drank in the past 6 months } \\
\hline No & 183 & $54 \cdot 3$ & $1 \cdot 00$ & & 74 & $25 \cdot 6$ & $1 \cdot 00$ & \\
\hline Yes & 99 & $59 \cdot 3$ & $1 \cdot 12(0 \cdot 74-1 \cdot 69)$ & 0.597 & 57 & $26 \cdot 5$ & $1.09(0 \cdot 71-1 \cdot 69)$ & 0.694 \\
\hline \multicolumn{9}{|c|}{ Used illicit drugs in the past 6 months } \\
\hline No & 171 & $53 \cdot 8$ & $1 \cdot 00$ & & 97 & $25 \cdot 3$ & $1 \cdot 00$ & \\
\hline Yes & 111 & $59 \cdot 7$ & $1 \cdot 36(0 \cdot 91-2 \cdot 01)$ & $0 \cdot 132$ & 34 & $28 \cdot 1$ & $1 \cdot 21(0 \cdot 75-1 \cdot 95)$ & $0 \cdot 440$ \\
\hline \multicolumn{9}{|l|}{$\mathrm{CD}^{+}{ }^{+} \mathrm{T}$ cell count $(\mathrm{cells} / \mu \mathrm{l})$} \\
\hline$\geqslant 350$ & 104 & $50 \cdot 5$ & $1 \cdot 00$ & & - & - & - & - \\
\hline $200-349$ & 119 & $57 \cdot 2$ & $1 \cdot 31(0 \cdot 88-1 \cdot 95)$ & $0 \cdot 189$ & - & - & - & - \\
\hline$<200$ & 59 & $65 \cdot 6$ & $1 \cdot 79(1 \cdot 05-3 \cdot 05)$ & 0.031 & - & - & - & - \\
\hline
\end{tabular}

${ }^{\text {a }}$ Adjusted for all the other variables in the table.

and their associations with EBV DNA would not be biased seriously since HIV infection was determined by laboratory tests and questions on dietary were not sensitive. Last but not least, although we included all the HIV-infected MSM attending the clinic with matched controls during the study period, the prevalence and the association may not be representative for all MSM in Shanghai or in China.

In conclusion, this study reported a prevalence of $56 \%$ for positive whole blood EBV DNA among the 
HIV-infected MSM, higher than the prevalence of $26 \%$ among the HIV-negative MSM in Shanghai, China. It also reported significant association between HIV infection, frequent pickled, smoked, or salty food intake, and lower $\mathrm{CD} 4^{+} \mathrm{T}$ cell counts with detectable whole blood EBV DNA. These results suggest that HIV-positive MSM in Shanghai, China are at higher risk for EBV-related cancers and further conformational studies are warranted.

\section{ACKNOWLEDGEMENTS}

This study was supported by Natural Science Foundation of China (grant no. 81361120385), Shanghai Municipal Health and Family Planning Commission (grant numbers GWTD2015S05, 15GWZK0101, 15GWZK0801, and 201440328), and the Chinese National Major Science \& Technology Project of Infectious Diseases (grant no. 2012ZX10001007-006).

\section{DECLARATION OF INTEREST}

None.

\section{ETHICAL STANDARDS}

The authors assert that all procedures contributing to this work comply with the ethical standards of the relevant national and institutional committees on human experimentation and with the Helsinki Declaration of 1975, as revised in 2008.

\section{REFERENCES}

1. Antiretroviral Therapy Cohort Collaboration. Life expectancy of individuals on combination antiretroviral therapy in high-income countries: a collaborative analysis of 14 cohort studies. The Lancet 2008; 372: 293299. doi: 10.1016/S0140-6736(08)61113-7.

2. Pantanowitz L, Schlecht HP, Dezube BJ. The growing problem of non-AIDS-defining malignancies in HIV. Current Opinion in Oncology 2006; 18: 469-478. doi: 10.1097/01.cco.0000239886.13537.ed.

3. Cheung MC, Pantanowitz L, Dezube BJ. AIDS-related malignancies: emerging challenges in the era of highly active antiretroviral therapy. The Oncologist 2005; 10: 412-426. doi: 10.1634/theoncologist.10-6-412.

4. Engels EA. Non-AIDS-defining malignancies in HIVinfected persons: etiologic puzzles, epidemiologic perils, prevention opportunities. AIDS (London, England) 2009; 23: 875-885. doi: 10.1097/QAD.0b013 e328329216a.
5. Younas M, et al. Immune activation in the course of HIV-1 infection: causes, phenotypes and persistence under therapy. HIV Medicine 2016; 17: 89-105. doi: 10.1111/hiv. 12310 .

6. Ipp H, et al. Role of inflammation in HIV-1 disease progression and prognosis. Critical Reviews in Clinical Laboratory Sciences 2014; 51: 98-111. doi: 10.3109/ 10408363.2013.865702.

7. Wada N, et al. Cause-specific life expectancies after 35 years of age for human immunodeficiency syndromeinfected and human immunodeficiency syndromenegative individuals followed simultaneously in long-term cohort studies, 1984-2008. American Journal of Epidemiology 2013; 177: 116-125. doi: 10.1093/aje/kws321.

8. Cobucci RNO, et al. Assessing the impact of HAART on the incidence of defining and non-defining AIDS cancers among patients with HIV/AIDS: a systematic review. Journal of Infection and Public Health 2015; 8: 1-10. doi: 10.1016/j.jiph.2014.08.003.

9. Silverberg MJ, et al. Cumulative incidence of cancer among HIV-infected individuals in North America. Annals of Internal Medicine 2015; 163: 507-518. doi: 10.7326/M14-2768.

10. Amon W, Farrell PJ. Reactivation of Epstein-Barr virus from latency. Reviews in Medical Virology 2005; 15: 149-156. doi: 10.1002/rmv.456.

11. Punnett A, Tsang RW, Hodgson DC. Hodgkin lymphoma across the age spectrum: epidemiology, therapy, and late effects. Seminars in Radiation Oncology 2010; 20: 30 -44. doi: 10.1016/j.semradonc.2009.09.006.

12. Pietersma F, Piriou E, van Baarle D. Immune surveillance of EBV-infected B cells and the development of non-Hodgkin lymphomas in immunocompromised patients. Leukemia \& Lymphoma 2008; 49: 1028-1041. doi: 10.1080/10428190801911662.

13. Molyneux EM, et al. Burkitt's lymphoma. The Lancet 2012; 379: 1234-1244. doi: 10.1016/S0140-6736(11) 61177-X.

14. Busson P, et al. EBV-associated nasopharyngeal carcinomas: from epidemiology to virus-targeting strategies. Trends in Microbiology 2004; 12: 356-360. doi: 10 . 1016/j.tim.2004.06.005.

15. Chua MLK, et al. Nasopharyngeal carcinoma. The Lancet 2016; 387: 1012-1024. doi: 10.1016/S0140-6736 (15)00055-0.

16. Guo X, et al. Evaluation of nonviral risk factors for nasopharyngeal carcinoma in a high-risk population of Southern China. International Journal of Cancer 2009; 124: 2942-2947. doi: 10.1002/ijc.24293.

17. Xue W-Q, et al. Quantitative association of tobacco smoking with the risk of nasopharyngeal carcinoma: a comprehensive meta-analysis of studies conducted between 1979 and 2011. American Journal of Epidemiology 2013; 178: 325-338. doi: 10.1093/aje/ kws479.

18. National Cancer Institute. Adult Hodgkin Lymphoma Treatment (PDQ ${ }^{\circledR}$ ). 2016: (https://www.cancer.gov/ types/lymphoma/patient/adult-hodgkin-treatment-pdq\# link/_1). 
19. Chihara D, et al. New insights into the epidemiology of non-Hodgkin lymphoma and implications for therapy. Expert Review of Anticancer Therapy 2015; 15: 531544. doi: 10.1586/14737140.2015.1023712.

20. Han B-L, et al. Systematic review on Epstein-Barr virus (EBV) DNA in diagnosis of nasopharyngeal carcinoma in Asian populations. Asian Pacific Journal of Cancer Prevention 2012; 13: 2577-2581.

21. Du X-J, et al. Circulating EBV DNA, globulin and nodal size predict distant metastasis after intensitymodulated radiotherapy in stage II nasopharyngeal carcinoma. Journal of Cancer 2016; 7: 664-670. doi: $10.7150 /$ jca. 14183 .

22. Lei KIK, et al. Quantitative analysis of circulating cellfree Epstein-Barr virus (EBV) DNA levels in patients with EBV-associated lymphoid malignancies. British Journal of Haematology 2000; 111: 239-246. doi: 10.1111/j.1365-2141.2000.02344.x.

23. Park JH, et al. Pretreatment whole blood Epstein-Barr virus-DNA is a significant prognostic marker in patients with Hodgkin lymphoma. Annals of Hematology 2016; 95: 801-808. doi: 10.1007/s00277-016-2610-5.

24. van Baarle D, et al. High prevalence of Epstein-Barr virus type 2 among homosexual men is caused by sexual transmission. The Journal of Infectious Diseases 2000; 181: 2045-2049. doi: 10.1086/315521.

25. Yang $\mathbf{J}$, et al. Prevalence and mortality of cancer among HIV-infected inpatients in Beijing, China. BMC Infectious Diseases 2016; 16: 82. doi: 10.1186/ s12879-016-1416-3.

26. National Health and Family Planning Commission of the People's Republic of China. 2015 China AIDS Response Progress Report. 2015 (http://unaids.org.cn/pics/ 20160614144959.pdf)

27. Aitken C, et al. Heterogeneity within the Epstein-Barr virus nuclear antigen 2 gene in different strains of Epstein-Barr virus. The Journal of General Virology 1994; 75: 95-100. doi: 10.1099/0022-1317-75-1-95.

28. Parra B, Slots J. Detection of human viruses in periodontal pockets using polymerase chain reaction. Oral Microbiology and Immunology 1996; 11: 289-293. doi: 10.1111/j.1399-302X.1996.tb00183.x.

29. Wang W-Y, et al. Consistent sequence variation of Epstein-Barr virus nuclear antigen 1 in primary tumor and peripheral blood cells of patients with nasopharyngeal Carcinoma. Clinical Cancer Research 2002; 8: 2586-2590.
30. van Kooij B, et al. Sequence analysis of EBV DNA isolated from mouth washings and PBMCs of healthy individuals and blood of EBV-LPD patients. Journal of Clinical Virology 2003; 28: 85-92. doi: 10.1016/S13866532(02)00269-X.

31. Wu C, et al. Results of detection of Epstein-Barr virus DNA in peripheral blood of HIV/AIDS patients. China Tropical Medicine (in Chinese) 2012; 12: 609-611.

32. Wang C, Chen Y, He Y. Study on changes of EpsteinBarr in HIV infected patients after highly active antiretroviral therapy. Journal of Medical Forum (in Chinese) 2008; 29: 4-7.

33. Rubinstein PG, Aboulafia DM, Zloza A. Malignancies in HIV/AIDS: from epidemiology to therapeutic challenges. AIDS (London, England) 2014; 28: 453-465. doi: 10.1097/QAD.0000000000000071.

34. Petrara MR, et al. Epstein-Barr virus load and immune activation in human immunodeficiency virus type 1-infected patients. Journal of Clinical Virology 2012; 53: 195-200. doi: 10.1016/j.jcv.2011.12.013.

35. Navarro J-T, et al. Plasma Epstein-Barr viral load measurement as a diagnostic marker of lymphoma in HIVinfected patients. Medicina Clinica (Barc) 2010; 135: 485-490. doi: 10.1016/j.medcli.2010.02.041.

36. Fan H, et al. Epstein-Barr viral load as a marker of lymphoma in AIDS patients. Journal of Medical Virology 2005; 75: 59-69. doi: 10.1002/jmv.20238.

37. Tisi MC, et al. Whole blood EBV-DNA predicts outcome in diffuse large B-cell lymphoma. Leukemia \& Lymphoma 2016; 57: 628-634. doi: 10.3109/10428194. 2015.1072766.

38. Piriou ER, et al. Altered EBV viral load setpoint after HIV seroconversion is in accordance with lack of predictive value of EBV load for the occurrence of AIDS-related non-Hodgkin lymphoma. Journal of Immunology (Baltimore, Md.: 1950) 2004; 172: 69316937.

39. Ho JHC, Huang DP, Fong YY. Salted fish and nasopharyngeal carcinoma in southern Chinese. The Lancet 1978; 312: 626. doi: 10.1016/S0140-6736(78)92844-1.

40. Ning $\mathbf{J}-\mathbf{P}$, et al. Consumption of salted fish and other risk factors for Nasopharyngeal Carcinoma (NPC) in Tianjin, a low-risk region for NPC in the People's Republic of China. Journal of the National Cancer Institute 1990; 82: 291-296. doi: 10.1093/jnci/ 82.4.291. 